\title{
Maximizing Differentiated Throughput in IEEE 802.11e Wireless LANs
}

\author{
Jongwon Yoon, Sangki Yun, Hyogon Kim, and Saewoong Bahk $\dagger$ \\ Department of Computer Science and Engineering, Korea University \\ \{yoonj, lockdown99, hyogon\}@korea.ac.kr \\ †School of Electrical Engineering and Computer Science, INMC, Seoul National University \\ sbahk@snu.ac.kr
}

\begin{abstract}
The throughput performance of the Distributed Coordination Function (DCF) of the IEEE 802.11 MAC protocol quickly degrades as the number of contending stations increases. To solve this problem, it has been shown recently that adaptive contention window modulation based on channel idle time tracking can be used, generating near optimal throughput. In this paper, we extend the approach for the IEEE 802.11e network, where different QoS classes are defined. We show how to find the class-specific optimal contention window sizes that yield the maximum aggregate throughput while maintaining the target throughput difference between classes.
\end{abstract}

\section{Introduction}

It is well known that the throughput performance under IEEE 802.11 contention-based medium access called the Distributed Coordinated Function (DCF) [1] significantly degrades as the number of contending stations increases. This suboptimality in congestion resolution has been a focus of attention in recent studies, and the proposals from the studies can be largely classified into two categories. The first is reactively modifying the minimum contention window size $\left(C W_{\min }\right)$ upon transmission success and failure $[2,3]$. The second is analytically deriving the optimal $C W_{\min }$ for the given congestion level on the link and steering the system towards it $[4,5,6]$.

Algorithmic or analytical, the prior work on 802.11 throughput maximization are all centered around the idea of $C W_{\min }$ parameter modulation. This is because among all parameters that can be utilized to control the 802.11 medium access control (MAC) behavior, $C W_{\text {min }}$ has provably predictable dynamics [7]. However, an issue with the prior work is that they are for the legacy 802.11 links where modulating $C W_{\min }$ is outside the provisions of the protocol specification. Fortunately, the functionality is fully supported in the new standard 802.11e [8].

Although the IEEE 802.11e supports the $C W_{\min }$ modulation, the existing ideas to maximize the MAC-layer throughput is not directly applicable. This is because there are 4 access classes (ACs) in 802.11e, and they are bound to receive different $C W_{\min }$ values for QoS differentiation [8]. Therefore, there is an issue how to maximize the aggregate throughput of the 802.11 link while creating the QoS differentiation. In this paper, we solve this problem, and show how to set the $C W_{\min }$ values for each $\mathrm{AC}$, given the throughput differentiation targets.

In this paper, we only use $C W_{\min }$ parameter both to create throughput differentiation and to achieve the maximum aggregate throughput, for its provable predictability [7]. Notice there are other parameters in so called the EDCA parameter set in the 802.11e that we can use to steer the MAC-layer dynamics such as the maximum contention window size $\left(C W_{\max }\right)$, transmission opportunity limit $(T X$ OPlimit) and arbitration inter-frame sequences (AIFS's) [8]. However, their effects [9] are rather difficult to characterize quantitatively. So we let these parameters equal for all AC's in this paper, which we believe is acceptable as long as the intended throughput differentiation among the ACs is created with the $C W_{\min }$ parameter.

\section{Determining Per-class Optimal $C W_{\min }$}

In this section, we discuss how to obtain the per-class $C W_{\min }$ value set that serves a double purpose: yield predefined throughput ratio between terminals in different classes, and maximize the aggregate throughput of the given 802.11e link on which the terminals contend for bandwidth. To this aim, we proceed as follows. First, we briefly show that the ratio of $C W_{\min }$ 's is inversely proportional to the ratio of throughput between classes. Namely, we show for 
classes $i$ and $j(1 \leq i, j \leq K)$

$$
\frac{C W_{\min }^{(i)}}{C W_{\min }^{(j)}}=\frac{\Theta^{(j)}}{\Theta^{(i)}}
$$

where $\Theta^{(l)}$ is the throughput of a terminal in class $l$. The aggregate throughput is given by the sum of the per-class throughputs, i.e., $\Theta=\sum_{l} \Theta^{(l)} n^{(l)}$, where $n^{(l)}$ is the population in class $l$. Second, we obtain the vector $W^{*}=$ $\left(C W_{\min }^{(1)}, C W_{\min }^{(2)}, \ldots, C W_{\min }^{(K)}\right)$ that generates the maximum aggregate throughput for the entire $802.11 \mathrm{e}$ wireless LAN. Namely, for the vector $W^{*}$ we require

$$
\forall_{W^{\prime} \in \mathcal{W}} \quad \Theta\left(W^{\prime}\right) \leq \Theta\left(W^{*}\right)
$$

where $\mathcal{W}$ is the set of feasible $C W_{\min }$ assignments to the 802.11e classes.

We remark that Equation (1) does not automatically lead to throughput maximization that is required by the condition Equation (2). For instance, $W_{1}=(1,2,4,8)$ will lead to the same bandwidth distribution with $W_{2}=$ $(32,64,128,256)$, but the aggregate throughput for these two $C W_{\min }$ vectors can be totally different. As a matter of fact, the very contribution of this paper is to show how we get the vector $W^{*}$ that simultaneously achieves both Equation (1) and Equation (2). We also remark that the proposed scheme does not have to know the class population $n^{(l)}$ to determine $W^{*}$. This property is important, since the information is tricky to obtain in practice. Below, we discuss the details of the proposed solution.

\subsection{Generating Target Throughput Ratio Between Classes}

The fact that the ratio of $C W_{\min }$ 's between two classes of terminals leads to the inverse ratio of throughputs (i.e., Equation (1)) has been independently established by Yang et al. [7] and our previous work [10]. Here, we briefly recap the result in [10]. Assuming the same average packet size $E[P]$ for each class, the throughput ratio is given as:

$$
\frac{\Theta^{(j)}}{\Theta^{(i)}}=\frac{\frac{E[P]}{T^{(j)}}}{\frac{E[P]}{T^{(i)}}}=\frac{T^{(i)}}{T^{(j)}}
$$

where $T^{(l)}$ is the average time that takes a terminal in class $l$ to successfully transmit a frame. It consists of the collision, successful transmission, idle (including CSMA/CA backoff), and the channel time occupied by other terminals transmission [10]. Namely,

$$
\begin{aligned}
T^{(l)} & =c^{(l)} E\left[T_{c}^{(l)}\right]+T_{s}^{(l)}+\left(c^{(l)}+1\right) E\left[T_{i}^{(l)}\right] \\
& +\sum_{k \neq l}^{M} \frac{P_{s}^{(k)}}{P_{s}^{(l)}}\left(T_{s}^{(k)}+c^{(k)} E\left[T_{c}^{(k)}\right]\right)
\end{aligned}
$$

where $c^{(l)}$ is the average number of collisions per transmission for class $l$ terminals. $P_{s}^{(l)}$ is the transmission success probability for class $l$, and $T_{c}^{(l)}, T_{s}^{(l)}, T_{i}^{(l)}$ are collision, successful transmission and idle times, for class $l$, respectively. Let $C W_{\min }^{(i)}=C W_{\min }^{(j)} \cdot \frac{j}{i}$. Then, substituting the $T^{(l)}$ in Equation (3), and after some approximations, [10] shows

$$
\begin{aligned}
\frac{\Theta^{(i)}}{\Theta^{(j)}} & \approx \frac{i}{j} \cdot \frac{j\left(C+T_{s}^{(j)}\right)+i\left(C+T_{s}^{(i)}\right)+j D^{(j)}}{i\left(C+T_{s}^{(i)}\right)+j\left(C+T_{s}^{(j)}\right)+j D^{(j)}} \\
& =\frac{i}{j}=\frac{C W_{\text {min }}^{(j)}}{C W_{\text {min }}^{(i)}}
\end{aligned}
$$

where $C=c^{(l)} E\left[T_{c}^{(l)}\right]$ and $D=\left(c^{(l)}+1\right) E\left[T_{i}^{(l)}\right]$.

\subsection{Obtaining Multi-class Optimal $C W_{\min }$ Vector}

In order to obtain the optimal vector $W^{*}$ in this section, we proceed as follows. First, we show that the channel idle time can be represented by the number of contending stations $n$ [5]. Given $n$, it is straightforward to derive the optimal $C W_{\min }^{*}$ that yields the maximum throughput [11]. But notice $n$ here is not the real population in the $802.11 \mathrm{e}$ WLAN. Rather, it represents the number of contending terminals if there were a single class. In essence, we need to provide a way to derive the vector $W^{*}$ from the uni-class scalar $C W_{\text {min }}^{*}$, which we discuss below.

First of all, from the idle time $T_{i}$ in the 802.11e class, we derive the virtual population $n$, which we define to be the number of the single-class population that would lead to the same $T_{i}$ in the legacy 802.11 WLAN. Then we will get $C W_{\text {min }}^{*}$ from $n$. Notice that the probability of an idle slot in the single-class (legacy) 802.11 networks is given as:

$$
P_{i}=(1-\tau)^{n}
$$

where $\tau$ is the transmission probability in a given slot from a terminal in the virtual population $n$. The idle time is then given by:

$$
\begin{aligned}
T_{i} & =\frac{1}{1-P_{i}}-1 \\
& =\frac{1}{1-(1-\tau)^{n}}-1
\end{aligned}
$$

Since the conditional collision probability and the transmission probability are related as follows,

$$
p=1-(1-\tau)^{n-1}
$$

where

$$
\tau=1-(1-p)^{\frac{1}{n-1}}
$$


The idle time is a function of both $n$ and $p$ as given by:

$$
T_{i}=\frac{1}{1-(1-p)^{\frac{n}{n-1}}}-1 .
$$

And from Bianchi [11] we know that $p$ itself is a function of $n$, i.e.,

$$
n=1+\frac{\log (1-p)}{\log \left(1-\frac{2(1-2 p)}{(1-2 p)(w+1)+p \cdot w\left(1-(2 p)^{m}\right)}\right)}
$$

where $w=C W_{\min }$ and $m$ is the maximum backoff stage. Combining Equation (6) and (7), we can eliminate $p$, and obtain $T_{i}$ as a function of $n$, which can be numerically computed. Figure 1 shows the relation between $T_{i}$ and $n$ for $C W_{\min }=31$ (IEEE $802.11 \mathrm{~b}$ default), and the analytical derivation closely fits with the simulation of the IEEE 802.11 in ns-2 [12]. According to this result, we can find the number of contending stations in the network by observing the idle time.

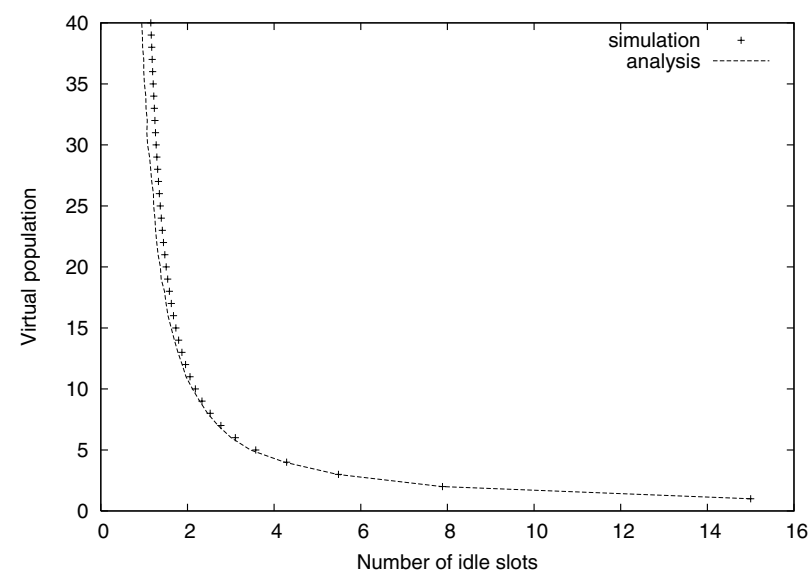

Figure 1. Number of stations vs. number of idle slots in the single-class 802.11b.

From the perspective of the 802.11e WLAN, the virtual population $n$ above obtained from trakcing $T_{i}$ can be considered to be the weighted sum of the class populations. Namely,

$$
n=\sum_{k} n^{(k)} \cdot \frac{\tau^{(k)}}{\tau^{(l)}}
$$

where $n^{(k)}$ is the number of stations and $\tau^{(k)}$ is the target transmission rate for class $k$, respectively. Specifically, the virtual population is the hypothetical number of class$l$ wireless terminals that would yield the total transmission rate $\tau$ in the single-class network (with class $l$ only). Here, $l$ is what we call the reference class, which can be any class. For convenience, we let it be the lowest-throughput class in this paper.
In addition to the nice (inverse) proportionality property in Equation (1), we establish here the relation between the ratios of $\tau$ 's and that of $C W_{\min }$ 's:

$$
\frac{\tau^{(l)}}{\tau^{(k)}} \approx \frac{C W_{\min }^{(k)}}{C W_{\min }^{(l)}} .
$$

From the legacy 802.11 , we know that the transmission probability $\tau$ is [13]:

$$
\tau=\frac{2}{1+C W_{\min }+p \cdot C W_{\min } \cdot \sum_{i=0}^{m-1}(2 p)^{i}} .
$$

Note that $\tau$ is a function of a single parameter, $p$, the conditional collision probability in a given slot. The ratio between the $\tau$ 's in the $802.11 \mathrm{e}$ link is then

$$
\frac{\tau^{(l)}}{\tau^{(k)}}=\frac{1+C W_{\min }^{(k)}+p^{(k)} \cdot C W_{\min }^{(k)} \sum_{i=0}^{m}\left(2 p^{(k)}\right)^{i}}{1+C W_{\min }^{(l)}+p^{(l)} \cdot C W_{\min }^{(l)} \sum_{i=0}^{m}\left(2 p^{(l)}\right)^{i}}
$$

Above, we can show that $p^{(l)} \approx p^{(k)}, l \neq k$ since for any class $i$

$$
\begin{aligned}
p^{(i)} & =1-\left(1-\tau^{(i)}\right)^{n^{(i)}-1} \prod_{k \neq i}\left(1-\tau^{(k)}\right)^{n^{(k)}} \\
& \approx 1-\left(1-\tau^{(i)} n^{(i)}+\tau^{(i)}\right) \prod_{k \neq i}\left(1-\tau^{(k)} n^{(k)}\right) \\
& \approx 1-\prod_{k}\left(1-\tau^{(k)} n^{(k)}\right)
\end{aligned}
$$

which is independent of the class $i$, assuming $n^{(k)}$ is large and $\tau^{(k)} \ll 1$. Replacing class-specific collision probabilities with a class-independent probability, i.e., $\forall_{k} p=p^{(k)}$, we get

$$
\begin{aligned}
\frac{\tau^{(l)}}{\tau^{(k)}} & \approx \frac{1+C W_{\min }^{(k)}+p \cdot C W_{\min }^{(k)} \cdot \frac{1}{1-2 p}}{1+C W_{\min }^{(l)}+p \cdot C W_{\min }^{(l)} \cdot \frac{1}{1-2 p}} \\
& =\frac{\frac{1-2 p}{1-p}+C W_{\min }^{(k)}}{\frac{1-2 p}{1-p}+C W_{\min }^{(l)}} \\
& \approx \frac{C W_{\min }^{(k)}}{C W_{\min }^{(l)}}
\end{aligned}
$$

since $\frac{1-2 p}{1-p}<1$ and $C W_{\min }^{(i)} \gg 1$ for all $i$. Therefore, like the throughput ratio, the ratio of transmission rates of different class stations is inversely proportional to the ratio of $C W_{\min }$ value.

Let $\tau$ be the total transmission probability in any given slot in the 802.11e WLAN. Then it is the sum of all transmission probabilities from all wireless terminals:

$$
\tau=\sum_{k} n^{(k)} \cdot \tau^{(k)} .
$$


The aggregate throughput $\Theta$ is given by [13]:

$$
\Theta=\frac{P_{s} P_{t r} E[P]}{\left(1-P_{t r}\right) \delta+P_{t r} P_{s} T_{s}+P_{t r}\left(1-P_{s}\right) T_{c}}
$$

where $E[P]$ is the average packet payload size, $P_{s}$ is the successful transmission probability and $P_{t r}$ is the probability that there is at least one transmission in a given slot. They are

$$
\begin{aligned}
P_{t r} & =1-(1-\tau)^{n}, \\
P_{s} & =n \tau(1-\tau)^{n} .
\end{aligned}
$$

And $T_{s}, T_{c}$ and $\delta$ respectively denote the duration of a successful transmission, average time the channel is sensed busy by each station during a collision and the duration of channel idle time. Note that the throughput $\Theta$ is a function of $n$ and $\tau$. In order to find the optimal $\tau$ that maximizes $\Theta$, we require

$$
\frac{\partial \Theta}{\partial \tau}=0 .
$$

Substituting Equation (11) for $\tau$, we notice

$$
\begin{aligned}
\frac{\partial \Theta}{\partial \tau} & =\frac{\partial \Theta}{\partial \sum_{k} n^{(k)} \cdot \tau^{(k)}} \\
& =\frac{\partial \Theta}{\partial \sum_{k} n^{(k)} \cdot \frac{C W_{\text {min }}^{(l)}}{C W_{\min }^{(k)}} \cdot \tau^{(l)}}
\end{aligned}
$$

where $\sum_{k} n^{(k)} \cdot \frac{C W_{m i n}^{(l)}}{C W_{\min }^{(k)}}$ is independent of $\tau^{(l)}$. Notice here that $\frac{C W_{\min }^{(l)}}{C W_{\min }^{(k)}}$ is a constant prescribed by the target throughput ratio, as shown in Section II-A. Therefore, we can reduce Equation (12) to

$$
\frac{\partial \Theta}{\partial \tau^{(l)}}=0
$$

By solving Equation (13) we can obtain the optimal transmission rate $\tau^{*(l)}$ that maximizes the aggregate throughput $\Theta$. Once we have $n$ and $\tau^{*(l)}$, we can get the $p^{*(l)}$ from Equation (4). Then exploiting the relation between the transmission rate and the collision probability, we get $C W_{\min }^{*(l)}$. Specifically, we use Equation (4), (5), and (10) to get $C W_{m i n}^{*(l)}$.

From Bianchi [11] we know that the transmission probability $\tau$ itself is a function of $p$ and $w$ i.e.,,

$$
\tau=\frac{2(1-2 p)}{(1-2 p)(w+1)+p w\left(1-(2 p)^{m}\right)}
$$

where $w=C W_{\min }$ and $m$ is the maximum backoff stage. By modifying Equation (14) with $C W_{\min }$, we can get

$$
C W_{\text {min }}^{*(l)}=\frac{2\left(1-2 p^{*(l)}\right)-\left(1-2 p^{*(l)}\right) \tau^{*(l)}}{\left(1-2 p^{*(l)}+p^{*(l)}\left(1-\left(2 p^{*(l)}\right)^{m}\right)\right) \tau^{*(l)}} .
$$

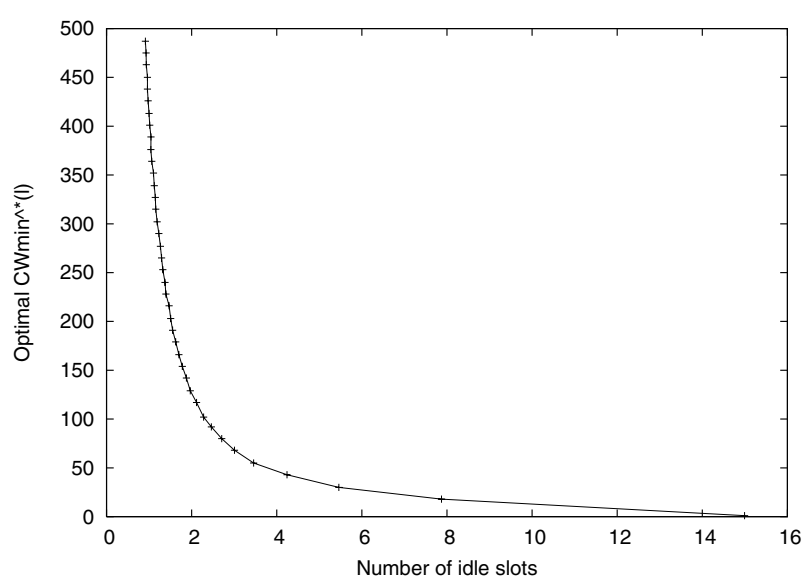

\section{Figure 2. Optimal $C W_{\text {min }}^{*(l)}$ for the reference class $l$ vs. channel idle time.}

Plugging in $p^{*(l)}$ and $\tau^{*(l)}$ Equation (15), we get the $C W_{\min }^{*(l)}$. Figure 2 shows the relation between $T_{i}$ and optimal $C W_{\min }^{*(l)}$.

Finally, we can rely on Equation (1) to find the rest of the values for the optimal vector $W^{*}$, namely,

$$
C W_{\min }^{*(j)}=\frac{\Theta^{*(i)}}{\Theta^{*(j)}} \cdot C W_{\min }^{*(i)}
$$

where the target throughput ratio between classes is prescribed.

Since the idle time is locally observed by each station through the Clear Channel Assessment (CCA), a core functionality of the IEEE 802.11 PHY standard, a station in class $k$ can first estimate the optimal $C W_{\text {min }}^{*(l)}$ for the reference class $l$. With this information, it can determine its own $C W_{\min }^{*(k)}$ by Equation (16). No central control or explicit coordination between terminals is necessary. Although the analytical derivation is complicated, we can tabulate and store in the MAC firmware only the idle time $T_{i}$ and its corresponding $C W_{\text {min }}^{*(l)}$ for the reference class with sufficient quantization. Referring to the table will be a single memory read operation, instead of a lengthy computation.

\section{Performance Evaluation}

In this section, we validate the analysis through simulation experiments. We run UDP flows on the 802.11e QBSS for which we modify the legacy 802.11 module in the ns- 2 simulator [12]. The UDP flows fall into one of the throughput classes, so they receive different throughput ratio guarantees. We let the wireless terminals in the QBSS all in mutual transmission range and close and equi-distant to the QAP. 
For UDP, it takes on the average $1,880 \mu$ s to send one packet over a $802.11 \mathrm{~b}+\mathrm{e}$ link, with $11 \mathrm{Mbps}$ nominal bitrate. This is because a transmission goes through the following sequence: DIFS $(50 \mu \mathrm{s})+$ contention $(310 \mu \mathrm{s}$ on average $=15.5$ slots $)+802.11$ DATA $(1,308 \mu \mathrm{s})+\operatorname{SIFS}(10 \mu \mathrm{s})$ +802.11 ACK $(202 \mu \mathrm{s})$. Here, the 802.11 DATA frame carries the UDP packet with up to 1,500 bytes payload. The achievable average transport throughput is thus $6.383 \mathrm{Mbps}$. (Any higher throughput is obtainable only through the reduction in the contention idle time with increased number of terminals, which is then offset by the collision overhead.) All simulations run for 1,000 seconds and we iterate each simulation for 10 times to increase confidence.

\subsection{Optimality of $W^{*}$}

In this part, we corroborate through simulation the claim that the optimal $W^{*}$ in Equation (16) does generate the maximum aggregate throughput $\Theta^{*}$. We simulate three different scenarios and the specifications for each scenario are in Table 1.

Table 1. Three Scenarios

\begin{tabular}{|c|c|c|}
\hline Scenario & $\begin{array}{c}\text { Population } \\
\text { in classes (A:B:C) }\end{array}$ & $\begin{array}{c}\text { Throughput ratio } \\
\text { between classes (A:B:C) }\end{array}$ \\
\hline \hline I & $2: 2: 2$ & $4: 2: 1$ \\
\hline II & $4: 5: 6$ & $4: 2: 1$ \\
\hline III & $4: 4: 0$ & $2: 1: 0$ \\
\hline
\end{tabular}

In Scenario I, even though the number of stations is 6, the virtual population is 14 according to Equation (8). The optimal contention window size corresponding to this virtual population is $C W_{\min }^{*(A)}=38$ according to Equation (15) if $A$ is the reference class. Table 2 lists up $C W_{\text {min }}^{*(A)}$ for each scenario. The optimal contention window size for other classes can be easily obtained by scaling the reference value according to Equation (16).

Table 2. $n$ vs. $C W_{\min }^{*(A)}$
\begin{tabular}{|c|c|c|}
\hline Scenario & Virtual population $n$ & $C W_{\min }^{*(A)}$ \\
\hline \hline I & 14 & 38 \\
\hline II & 38 & 118 \\
\hline III & 12 & 70 \\
\hline
\end{tabular}

In Figure 3, we vary the $C W_{\min }^{(A)}$, and observe the aggregate throughput to verify that the aggregate throughput maximizes when the $C W_{\min }^{(A)}$ value is near the analytically obtained optimum $W_{(A)}^{*}$. While we vary $C W_{m i n}^{(A)}$, we also

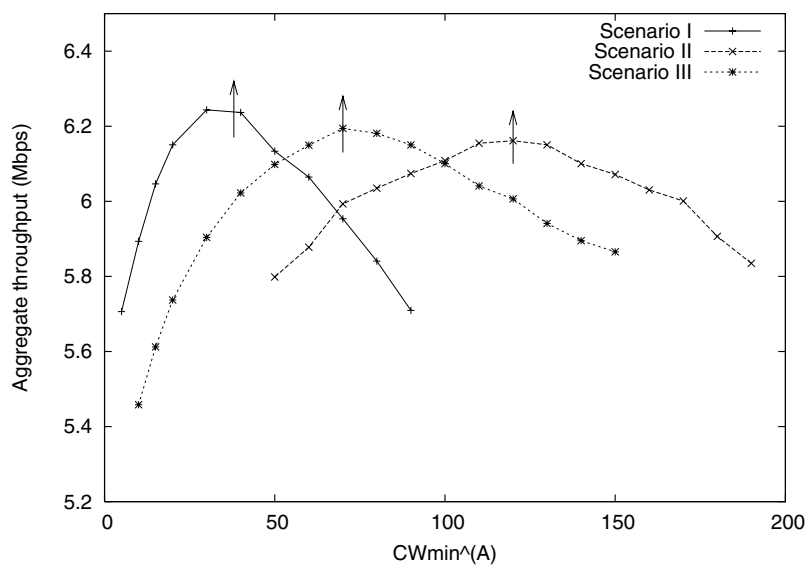

Figure 3. Aggregate throughput for each scenario.

keep the $C W_{\min }$ ratio for each class 1:2:4 to maintain the differentiation among the classes. For example, when the $C W_{\text {min }}^{(A)}=38$, we let $C W_{\text {min }}^{(B)}=76$ and $C W_{\text {min }}^{(C)}=152$. In the figure, the arrows mark whether the analysis predicts the maximum. We notice that the analysis is reasonably close to the real optimum.

\subsection{Differentiated Throughput}

Compared with the station with higher $C W_{\min }$ value, stations with a lower value of $C W_{\min }$ show higher throughput performance, because a lower value of $C W_{\min }$ will reduce the average backoff time before transmission attempts, correspondingly increasing the probability to grab the channel. Here we demonstrate that the simulation bears out Equation (1). We take Scenario I in Table 1, except that we vary the class population $n^{(l)}, l \in\{A, B, C\}$ between 1 to $10^{1}$ and evaluate the per-class throughput. If our method works properly, the ratio of $C W_{\min }$ 's should remain constant (while their absolute values adapt to the increased contention), and so should the throughput ratio.

Figure 4 compares the per-class aggregate throughput values. For example, consider the following case there are 3 stations in each class and the throughput ratio of the class is $4: 2: 1$. Then virtual population is 21 and the optimal $C W_{\min }$ value for the lowest class $\mathrm{A}$ is 61 . By Equation (16) the optimal $C W_{\min }$ value for class B and C is 122 and 244. Like this example, all stations adapt their $C W_{\min }$ value to maintain the differentiation throughput while the number of stations in each class increase.

We see that the ratio of the per-class throughput stays

\footnotetext{
${ }^{1}$ With 3 classes, the per-class population of 10 amounts to a total of 30 stations. The anecdotal capacity of IEEE $802.11 \mathrm{~b}$ is said to be 20 to 30 stations.
} 


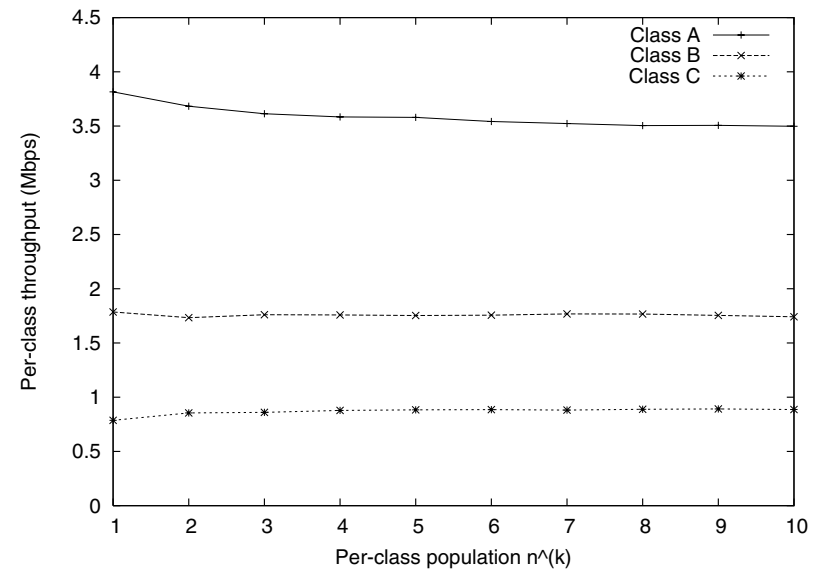

Figure 4. Per-class throughput.

close to $\Theta^{(A)}: \Theta^{(B)}: \Theta^{(C)}=4: 2: 1$. This is what we expect from prior works $[7,10]$. As the per-class population increases, however, the system begins to slightly lose the precise ratio especially for the highest class. We believe this stems from the increasing error in estimating $n$ from the idle time $T_{i}$, when $n$ is large so the contention is high (see Figure 1). The underestimation of the population hence contention level leads to smaller $C W_{\min }$ values, which is not enough to cope with the contention. This issue is under investigation, and will be followed up on in future work.

\subsection{Total Aggregate Throughput}

We remark that per-class population $n^{(k)}=10$ is equivalent to the total population of 30 in Figure 4, and it is around the maximum number of terminals that a $802.11 \mathrm{~b}$ WLAN is usually provisioned for. To the right of the vertical line, therefore, is the regime that involves severe contention. Nevertheless, we notice in Figure 4 that the total aggregate throughput $\left(\Theta=\sum_{k \in\{A, B, C\}} \Theta^{(k)}\right)$ holds relatively well under load variation. This is the result of the dynamic $C W_{\min }$ modulation based on $T_{i}$. Here, we evaluate the impact of the $C W_{\min }$ adaptation above and beyond the ratio maintenance. (i.e., through Equation (15) and Equation (16))

In this experiment, we again configure three classes $\mathrm{A}$, $\mathrm{B}$ and $\mathrm{C}$, and we set the $C W_{\min }$ ratio of the class to $C W_{\text {min }}^{(A)}: C W_{\text {min }}^{(B)}: C W_{\text {min }}^{(C)}=1: 2: 4$ for the differentiation. We compare two schemes, one with the ratio maintenance and adaptation, and the other with only ratio setting. For the latter, we fix $C W_{\min }$ values of keeping the $C W_{\min }$ ratio for each class 1:2:4 to maintain the differentiation among the classes.

From Figure 5 we see that aggregate throughput of standard scheme decreases as the number of stations increases. However, the adaptive $C W_{\min }$ scheme always performs

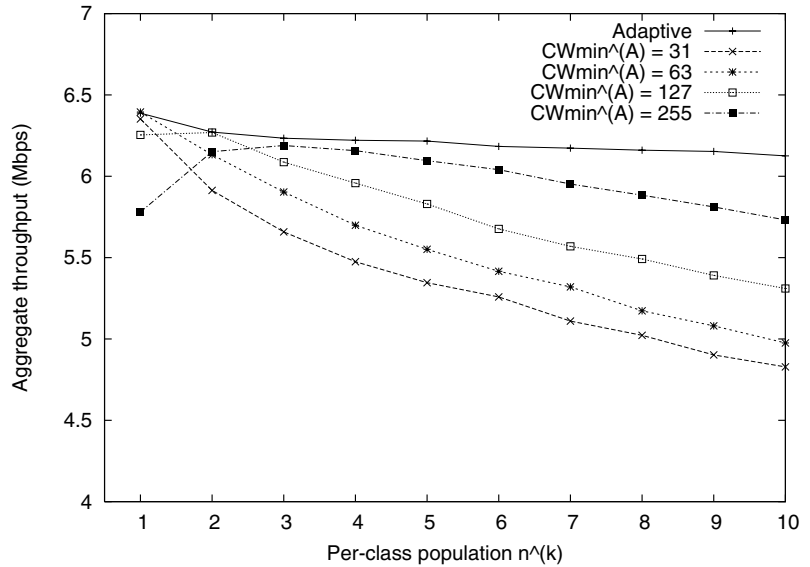

Figure 5. Aggregate throughput with and without dynamic adaptation.

better than any of the fixed schemes, and the throughput gap widens as the population increases. Again, the aggregate throughput of the adaptive scheme decreases with $n$, an evidence of contention underestimation in the severe congestion regime that we mentioned above.

\section{Conclusion}

We show that service differentiation and throughput maximization can be simultaneously achieved through appropriate assignments of per-class optimal $C W_{\min }$. By observing the number of idle slots between two consecutive transmission attempts through the IEEE 802.11 CCA functionality, we can estimate the number of contending stations, which is the virtual population of reference class (which can be any class) terminals. We use this information to derive the throughput-maximizing per-class transmission probability, and to obtain the optimal $C W_{\min }$ for the reference class. The $C W_{\min }$ for non-reference classes can be obtained by setting it inversely proportional to the target throughput ratio with respect to the reference class. By adjusting $C W_{\min }$ this way, we can simultaneously achieve service differentiation and aggregate throughput maximization in the IEEE 802.11e wireless LANs.

There are other parameters in so called the EDCA parameter that we can use to create throughput differentiation and to achieve the maximum aggregate throughput. The future researches include the optimization of EDCA parameters to get the performance improvement in the IEEE $802.11 \mathrm{e}$. 


\section{Acknowledgement}

This research is supported by the Ubiquitous Autonomic Computing and Network Project, the Ministry of Information and Communication (MIC) 21st Century Frontier R\&D Program in Korea.

\section{References}

[1] IEEE, Part 11: Wireless LAN Medium Access Control (MAC) and Physical Layer (PHY) Specifications. IEEE Std 802.11-1999, Aug. 1999.

[2] Q. Pang, S. C. Liu, J. Lee and S. H. Chan, "A TCP-like Adaptive Contention Window Scheme for WLAN," in proceedings of ICC 2004.

[3] Z. Haas and J. Deng, "On Optimizing the Backoff Interval for Random Access Schemes," IEEE Transactions on Communications, 51(12), Dec. 2003.

[4] F. Cali, M. Conti and E. Gregori, "Dynamic Tuning of the IEEE 802.11 Protocol," ACM/IEEE Transactions on Networking, 8(6), 2000.

[5] M. Heusse, F. Rousseau, R. Guillier and A. Duda, "Idle Sense: An Optimal Access Method for High Throughput and Fairness in Rate Diverse Wireless LANs," in proceedings of ACM Sigcomm, 2005.

[6] Y. Xiao, "Performance Analysis of Priority Schemes for IEEE 802.11 and IEEE 802.11e Wireless LANs,"
IEEE Transactions on Wireless Communications, 4(4), Jul. 2005.

[7] Y. Yang, J. Wang and R. Kravets, "Distributed Optimal Contention Window Control for Elastic Traffic in Wireless LANs," in proceedings of IEEE Infocom, 2005.

[8] ANSI/IEEE, "802.11e: Wireless Medium Access Control (MAC) and Physical Layer (PHY) Specifications: Medium Access Control (MAC) Enhancements for Quality of Serivce (QoS),” Nov. 2002.

[9] I. Aad and C. Castelluccia, "Differentiation Mechanism for IEEE 802.11," in proceedings of IEEE Infocom, 2001.

[10] H. Kim, S. Yun and I. Kang, "Resolving 802.11 Performance Anomalies through QoS Differentiation," IEEE Communications Letters, 9(7), Jul. 2005.

[11] G. Bianchi and I. Tinnirello, "Kalman Filter Estimation of the Number of Competing Terminals in an IEEE 802.11 Network," in proceedings of IEEE Infocom, 2003.

[12] The ns-2 Simulator. Available at http://www.isi.edu/nsnam/ns/.

[13] G. Bianchi, "Performance Analysis of the IEEE 802.11 Distributed Coordination Function," IEEE Journal on Selected Areas in Communications, 18(3), pp. 535 - 547, Mar. 2000. 